\title{
The PAMELA Satellite Experiment: An Observatory in Space for Particles, Antiparticles and Nuclei in the Cosmic Rays
}

\section{Ricci ${ }^{1}$ on behalf of the PAMELA Collaboration}

INFN, Laboratori Nazionali di Frascati, Via Enrico Fermi 40, I-00044 Frascati,(Roma) Italy marco.ricci@lnf.infn.it

\begin{abstract}
The instrument PAMELA, in orbit since June 2006 on board the Russian satellite Resurs DK1, is designed to study charged particles, antiparticles and nuclei in the cosmic rays, with a particular focus on the search for antimatter and signals of dark matter, the isotopic composition and the investigation of phenomena connected with Solar and Earth physics. A combination of a magnetic spectrometer and different detectors allows antiparticles (antiprotons and positrons) to be reliably identified from a large background of other charged particles. New results on the antiprotonto-proton and positron-to-all electron ratios over a wide energy range (1-100 GeV) are presented, together with the measurement of the light nuclear component (from hydrogen to oxygen) of galactic cosmic rays in the range $100 \mathrm{MeV} / \mathrm{n}-200 \mathrm{GeV} / \mathrm{n}$.
\end{abstract}

XLVIII International Winter Meeting on Nuclear Physics - BORMIO2010

Bormio, Italy

January 25-29 2010

\footnotetext{
$1 \quad$ Speaker
} 


\section{Introduction}

PAMELA (a Payload for Antimatter-Matter Exploration and Light nuclei Astrophysics), is a space experiment installed in a pressurized container attached to the Russian Resurs-DK1 Earth-observation satellite (mass: 7.6 Tons; height: $7.4 \mathrm{~m}$ ). It was launched into space on June $15^{\text {th }}, 2006$ by a Soyuz-U rocket from the Baikonur cosmodrome in Kazakhstan, and placed in a nearly-polar (inclination 70 ${ }^{\circ}$ ) low-Earth elliptical orbit at an altitude in the range $350-610 \mathrm{~km}$.

PAMELA has been designed to measure in detail the spectra of primary and secondary components of the cosmic radiation. Its major scientific goals are the search for primordial antimatter (antihelium) and the indirect detection of dark matter by means of the precise measurement of antiprotons and positrons spectra in cosmic rays, over the largest energy range ever achieved. The long term period of data taking (the three years of nominal mission have recently been extended to five) provides unprecedented statistics with no atmospheric overburden reducing the systematic uncertainties of previous measurements obtained by balloon-borne experiments. Other pursued objectives are the study of the cosmic ray production and propagation in our galaxy by precise measurements of the absolute fluxes of primary and secondary light nuclei, the monitoring of solar activity and cosmic rays solar modulation, the investigation of the interaction processes of cosmic-rays with the Earth's magnetosphere and the search for high energy electrons in order to discover local sources.

PAMELA is conducted by an international collaboration constituted of several INFN (Italian National Institute of Nuclear Physics) Divisions and Italian Universities, three Russian institutions (MEPhI and FIAN Lebedev in Moscow and IOFFE in St. Petersburg), the University of Siegen in Germany and the Royal Technical Institute in Stockholm, Sweden.

\section{The PAMELA apparatus}

The PAMELA apparatus is composed of several particle detectors, as shown in Fig. 1, from top to bottom: a time of flight system (ToF) (S1, S2, S3), a magnetic spectrometer, an anticoincidence system (CARD, CAT, CAS), an electromagnetic imaging calorimeter, a shower tail catcher scintillator (S4), and a neutron detector. 


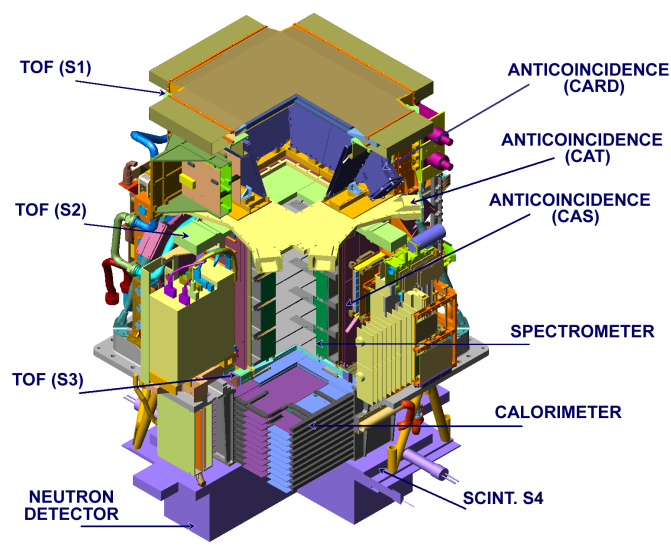

Figure 1: The PAMELA apparatus.

The core of the instrument is the magnetic spectrometer composed of a $0.43 \mathrm{~T}$ permanent magnet equipped with six planes of double-sided silicon detectors [1], each $300 \mu \mathrm{m}$ thick, that allows the sign, the absolute value of the charge and the rigidity of crossing charged particles to be determined. The spatial resolution of $4 \mu \mathrm{m}$ in the bending view assures a Maximum Detectable Rigidity (MDR) of $800 \mathrm{GV}$. Spillover effects limit the upper detectable antiparticle momentum to $190 \mathrm{GeV} / \mathrm{c}$ and to $270 \mathrm{GeV} / \mathrm{c}$ for antiprotons and positrons, respectively.

The discrimination between the leptonic and hadronic components is performed by the imaging calorimeter complemented by the neutron detector.

The calorimeter is composed by 44 ministrip silicon layers, $380 \mu \mathrm{m}$ thick, each interleaved with 22 tungsten planes for a depth of 16.3 radiation lengths and 0.6 interaction lengths [2].

The neutron detector is made of $36{ }^{3} \mathrm{He}$ counters inserted in $1 \mathrm{~cm}$ thick polyethylene moderator [3].

A set of 3 pairs of stripped plastic scintillators constitutes the PAMELA trigger and Timeof-Flight (ToF) [4]. The timing resolution of the ToF system allows albedo particles to be identified. An additional set of plastic scintillators in anticoincidence defines the acceptance of the particles inside the spectrometer [5].

The scintillator S4 is mounted under the calorimeter and helps in the identification of high-energy electrons.

PAMELA overall size is $130 \mathrm{~cm} \times 70 \mathrm{~cm} \times 70 \mathrm{~cm}$, corresponding to a geometric factor of $21.5 \mathrm{~cm}^{2} \mathrm{sr}$ (for $\mathrm{R}>1 \mathrm{GV}$ ), with a total mass of $470 \mathrm{~kg}$, and a maximum power consumption of $360 \mathrm{~W}$. A complete description of the subdetector components can be found in [6].

\section{Antiprotons and positrons}

Since July 2006, the PAMELA instrument is daily transmitting 16 Gigabytes of data through satellite telemetry to the Ground Segment placed in Moscow at the NTsOMZ Center [7]. The results presented here are based on the data-set collected between July 2006 and 
February 2008. More than $10^{9}$ triggers were accumulated during a total acquisition time of approximately 500 days.

Antiproton identification was based on the determination of the rigidity by the spectrometer and the properties of the energy deposit and interaction topology in the calorimeter. The calorimeter was used to reject electrons.

The antiproton-to-proton flux ratio [8] measured by the PAMELA experiment is shown in Fig. 2, compared with theoretical calculations assuming pure secondary production of antiprotons during the propagation of cosmic rays in the galaxy.

The PAMELA data are in excellent agreement with recent results from other experiments, as shown in Fig. 3. The ratio increases smoothly from about $2 \times 10^{-5}$ at a kinetic energy of about $1 \mathrm{GeV}$ and levels off at about $1 \times 10^{-4}$ for energies above $10 \mathrm{GeV}$.

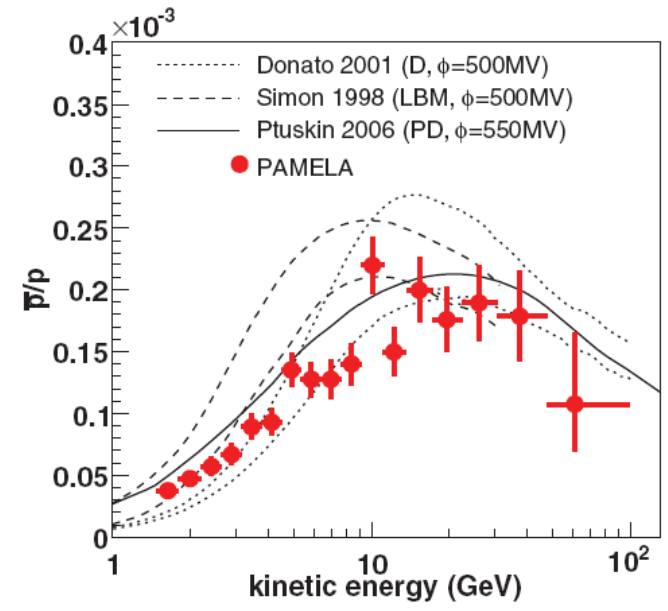

Figure 2: PAMELA antiproton-to-proton flux ratio compared with the theoretical calculations for a pure secondary production of antiprotons [9-11].

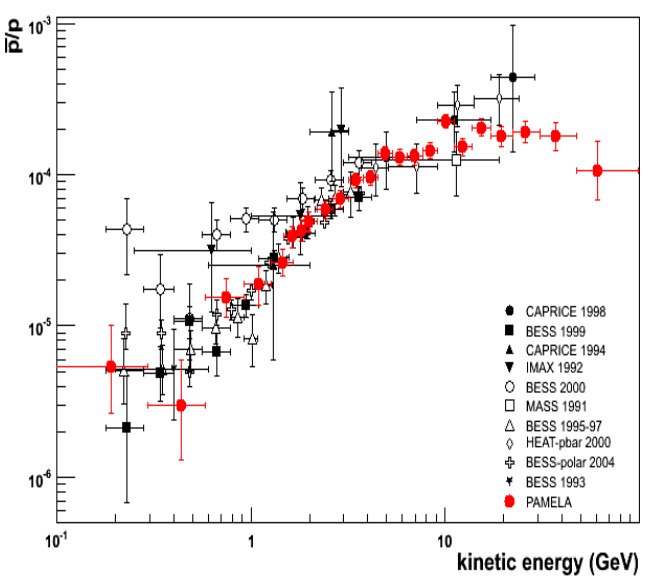

Figure 3: PAMELA antiproton-to-proton flux ratio compared with previous measurements [12].

The positron to all electron ratio [13] measured by the PAMELA experiment is given in Fig. 4. The calculation, shown in the same figure for pure secondary production of positrons during the propagation of cosmic rays in the Galaxy without reacceleration processes, proves that the positron fraction is expected to fall as a smooth function of increasing energy if secondary production dominates. In Fig. 5 the data are compared with other recent experimental measurements. 


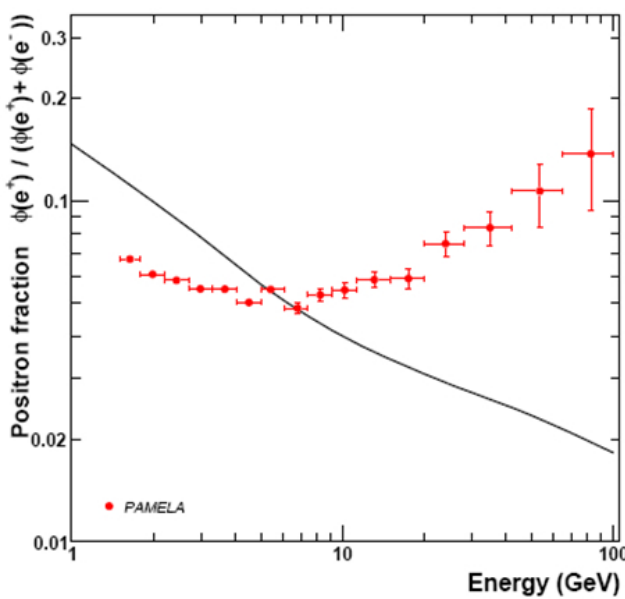

Figure 4: PAMELA positron fraction. The solid line shows a calculation for pure secondary positron production during the propagation of cosmic rays in the Galaxy [14].

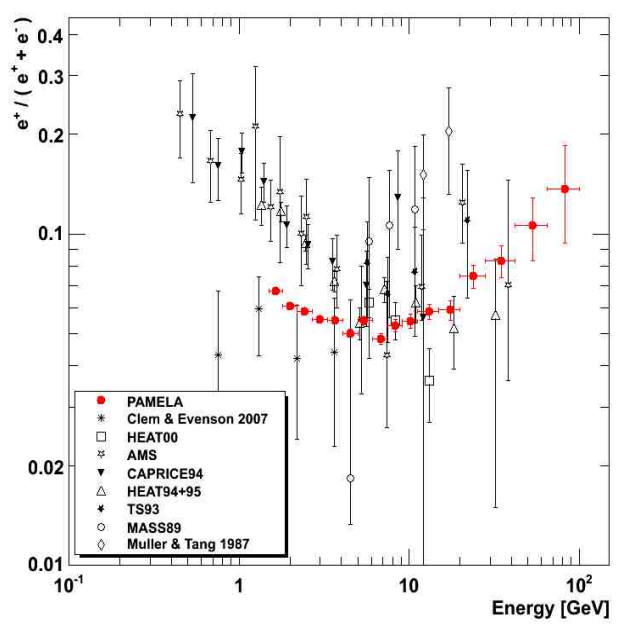

Figure 5: PAMELA positron fraction, compared with other recent experimental data [15].

Two features are clearly visible. At low energies, below $5 \mathrm{GeV}$, the PAMELA results are systematically lower than data collected during the 1990's (an effect due to solar modulation related to different periods of data taking), while at high energies, above $10 \mathrm{GeV}$, they show a positron fraction significantly increasing with energy. Between $5 \mathrm{GeV}$ and $10 \mathrm{GeV}$, the PAMELA positron fraction is compatible with other measurements. This excess of positrons in the range $10-100 \mathrm{GeV}$ has led to many speculations about its origin, as annihilation of dark matter, decaying dark matter, decaying of lightest superparticle dark matter, cosmic strings, young pulsars, nearby SNR (Supernova Remnants) (see, for a review, ref. [16]).

\section{Nuclei}

The nuclear component plays an important role in the study of cosmic rays. The relative abundances of the constituents of Galactic Cosmic Rays (GCRs) provide information about the cosmic ray transport mechanisms within the Galaxy. Cosmic rays of primary origin such as Carbon and Oxygen may interact with the interstellar medium to produce secondary fragments ( $\mathrm{Li}, \mathrm{Be}, \mathrm{B}$ ); the ratio of secondary to primary cosmic rays, being sensitive to the amount of material traversed by GCRs from the source to Earth, can be used as an input to constrain and check the propagation models. Furthermore, abundance ratios are less sensitive to instrumental uncertainties than absolute intensities.

PAMELA, mainly with the use of the tracker, the ToF and the first layers of the calorimeter, is able to measure the light nuclear component of cosmic rays (up to Oxygen) in the energy interval $100 \mathrm{MeV} / \mathrm{n}-200 \mathrm{GeV} / \mathrm{n}$. Preliminary results have been shown on B/C, Li/C, $\mathrm{Be} / \mathrm{C}$ and $\mathrm{C} / \mathrm{O}$. Here in Fig. 6, as an example, the $\mathrm{B} / \mathrm{C}$ ratio is shown compared to previous measurements. Analysis is in progress as far as more data and statistics are available to cover the whole energy range achievable. 


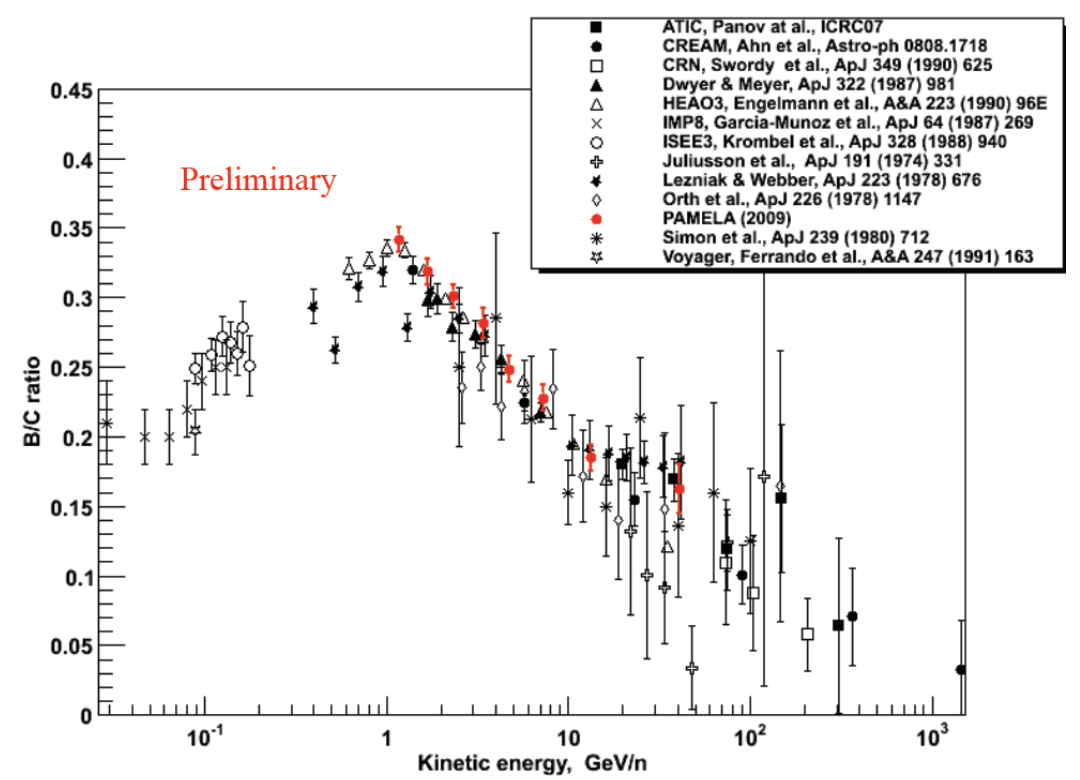

Figure 6: PAMELA preliminary $\mathrm{B} / \mathrm{C}$ ratio compared to previous measurements.

\section{References}

[1] O. Adriani et al., Nucl. Instr. Meth. A, 511, 72 (2003).

[2] M. Boezio et al., Nucl. Instr. Meth. A, 487, 407 (2002).

[3] Y. Stozhkov, Proc. $30^{\text {th }}$ Int. Cosmic Ray Conf., Merida (2007), vol. 2, p. 325.

[4] G. Osteria et al., Nucl. Instr. Meth. A, 535, 152 (2004).

[5] S. Orsi et al., Proc. $29^{\text {th }}$ Int. Cosmic Ray Conf., Pune (2005), vol. 3, p. 369.

[6] P.Picozza et al., Astrop. Phys., 27, 296 (2007).

[7] http://eng.ntsomz.ru

[8] O. Adriani et al., Phys. Rev. Lett., 102, 051101 (2009).

[9] M. Simon et al., Astrophys. Journal., 499, 250 (1998).

[10] F. Donato et al., Astrophys. Journal., 563, 172 (2001).

[11] V. S. Ptuskin et al., Astrophys. Journal., 642, 902 (2006).

[12] Experiments in the figure are cited in ref. [8].

[13] O. Adriani et al., Nature, 458, 607 (2009).

[14] I. V. Moskalenko and A. W. Strong, Astrophys. Journal., 493, 694 (1998).

[15] Experiments in the figure are cited in ref. [13].

[16] M. Boezio et al., New J. Phys. 11 (2009) 105023. 